\title{
ON THE DIMENSION OF THE GLOBAL SECTIONS OF THE ADJOINT BUNDLE FOR POLARIZED 5-FOLDS
}

\author{
Yoshiaki FUKUMA \\ (Received 6 April 2020 and revised 9 February 2021)
}

\begin{abstract}
Let $(X, L)$ denote a polarized manifold of dimension five. This study considers the dimension of the global sections of $K_{X}+m L$ with $m \geq 6$. In particular, we prove that $h^{0}\left(K_{X}+m L\right) \geq\left(\begin{array}{c}m-1 \\ 5\end{array}\right)$ for any polarized 5-fold $(X, L)$ with $h^{0}(L)>0$. Furthermore, we also consider $(X, L)$ with $h^{0}\left(K_{X}+m L\right)=\left(\begin{array}{c}m-1 \\ 5\end{array}\right)$ for some $m \geq 6$ with $h^{0}(L)>0$.
\end{abstract}

\section{Introduction}

Let $X$ be a smooth projective complex variety of dimension $n$, and let $L$ be an ample line bundle on $X$. Then, $(X, L)$ is called a polarized manifold. The adjoint bundle $K_{X}+m L$ of $(X, L)$ plays a key role in investigating $(X, L)$ (for example, see [2, Chapters 7, 9 and 11]), where $K_{X}$ and $m$ denote the canonical line bundle of $X$ and a natural number, respectively. For example, the nefness of $K_{X}+m L$ has been studied by numerous authors, and as a corollary, the non-negativity of the sectional genus, $g(X, L)$, of $(X, L)$ was obtained. In addition, we also note that numerous authors studied the base point freeness and very ampleness of adjoint bundles related to a conjecture of Fujita $[6,19,22,23]$.

Recently, the positivity of dimension $h^{0}\left(K_{X}+m L\right)$ has been discussed. For $m=n-1$, Beltrametti and Sommese proposed the following conjecture [2, Conjecture 7.2.7].

Conjecture 1. (Beltrametti-Sommese) Let $(X, L)$ be a polarized manifold with $\operatorname{dim} X=$ $n \geq 3$. Assume that $K_{X}+(n-1) L$ is nef. Then $h^{0}\left(K_{X}+(n-1) L\right)>0$.

For this conjecture, the following partial results have been obtained:

- In [11, Theorem 2.4] and [14, Theorem 3.1], the author proved that this conjecture is true if $n \leq 4$. (See also [3] and [4].) Besides, we also note that Andreatta and Fontanari [1] improved the result in [14].

- $\quad$ In [18, 1.2 Theorem], Höring proved that this conjecture is true if $h^{0}(L)>0$.

Moreover, we have classified $(X, L)$ for the following types in our previously conducted studies:

- $\quad$ Polarized 3-fold $(X, L)$ with $h^{0}\left(K_{X}+2 L\right) \leq 2[\mathbf{1 1}, \mathbf{1 3}]$.

- $\quad$ Polarized 4-fold $(X, L)$ with $h^{0}\left(K_{X}+3 L\right) \leq 1[\mathbf{1 4}, \mathbf{1 5}]$.

2010 Mathematics Subject Classification: Primary 14C20; Secondary 14J35.

Keywords: polarized manifold; adjoint bundles; Beltrametti-Sommese conjecture; sectional geometric genus. 
Additionally, we also considered the case where $m \geq n$. In [16, Conjecture 2], we proposed the following conjecture.

CONJECTURE 2. Let $(X, L)$ be an $n$-dimensional polarized manifold with $n \geq 3$.

(i) Then, $h^{0}\left(K_{X}+m L\right) \geq\left(\begin{array}{c}m-1 \\ n\end{array}\right)$ holds for every integer $m \geq n+1$. If equality holds for some $m \geq n+1$, then $(X, L) \cong\left(\mathbb{P}^{n}, \mathcal{O}_{\mathbb{P}^{n}}(1)\right)$.

(ii) If $h^{0}\left(K_{X}+n L\right)=0$, then $(X, L) \cong\left(\mathbb{P}^{n}, \mathcal{O}_{\mathbb{P}^{n}}(1)\right)$.

(iii) If $h^{0}\left(K_{X}+n L\right)=1$, then $(X, L)$ can be one of the following:

(iii.1) $(X, L) \cong\left(\mathbb{Q}^{n}, \mathcal{O}_{\mathbb{Q}^{n}}(1)\right)$;

(iii.2) $(X, L)$ is a scroll over a smooth elliptic curve with $L^{n}=1$.

It has been proved that Conjecture 2 is true only for the following cases:

- $\quad$ The case where $n \leq 4$ [11, Theorem 2.5; and 16, Theorem 3.1]. (See also [1, Theorem 8] for results concerning with Conjecture 2.)

- $\quad$ The case where $n \geq 5$ and $\operatorname{dim} \mathrm{Bs}|L| \leq 1$ for Conjecture 2(i) [16, Theorem 3.2(i)].

- $\quad$ The case where $n \geq 5$ and $h^{0}(L)>0$ for Conjecture 2(ii) and (iii) [16, Theorem 3.2(ii) and (iii)].

In this study, we consider Conjecture 2(i) for the case where $n=5$ and $h^{0}(L)>0$. Consequently, we prove that Conjecture 2(i) is true for this case.

Herein, we use the customary notation in algebraic geometry.

\section{Preliminaries}

Notation 2.1. Let $X$ be a projective variety of dimension $n$ and let $L$ be a line bundle on $X$. Then, we set

$$
\chi(t L)=\sum_{j=0}^{n} \chi_{j}(X, L)\left(\begin{array}{c}
t+j-1 \\
j
\end{array}\right) .
$$

Definition 2.1. [9, Definition 2.1] Let $X$ be a projective variety of dimension $n$ and let $L$ be a line bundle on $X$. For every integer $i$ with $0 \leq i \leq n$, the $i$ th sectional geometric genus $g_{i}(X, L)$ of $(X, L)$ is defined as follows:

$$
g_{i}(X, L)=(-1)^{i}\left(\chi_{n-i}(X, L)-\chi\left(\mathcal{O}_{X}\right)\right)+\sum_{j=0}^{n-i}(-1)^{n-i-j} h^{n-j}\left(\mathcal{O}_{X}\right) .
$$

Remark 2.1.

(i) Since $\chi_{n-i}(X, L) \in \mathbb{Z}$, we observe that $g_{i}(X, L)$ is integer by definition.

(ii) If $i=\operatorname{dim} X=n$, then $g_{n}(X, L)=h^{n}\left(\mathcal{O}_{X}\right)$.

(iii) If $i=0$, then $g_{0}(X, L)=L^{n}$.

(iv) If $i=1$, then $g_{1}(X, L)=g(X, L)$, where $g(X, L)$ denotes the sectional genus of $(X, L)$. If $X$ is smooth, then the sectional genus $g(X, L)$ can be given by

$$
g(X, L)=1+\frac{1}{2}\left(K_{X}+(n-1) L\right) L^{n-1} .
$$


(v) If $i=2$, then we obtain that (see $[10,(2.2 . \mathrm{A})]$ )

$$
\begin{aligned}
g_{2}(X, L)= & \frac{1}{12}\left(K_{X}+(n-1) L\right)\left(K_{X}+(n-2) L\right) L^{n-2}+\frac{1}{12} c_{2}(X) L^{n-2} \\
& +\frac{n-3}{24}\left(2 K_{X}+(n-2) L\right) L^{n-1}-1+h^{1}\left(\mathcal{O}_{X}\right) .
\end{aligned}
$$

(vi) If $i=3$, then we have (see [10, (2.2.B)])

$$
\begin{aligned}
g_{3}(X, L)= & \frac{(n-2)(n-3)^{2}}{48} L^{n}+\frac{(n-3)(3 n-8)}{48} K_{X} L^{n-1} \\
& +\frac{n-3}{24}\left(K_{X}^{2}+c_{2}(X)\right) L^{n-2}+\frac{1}{24} K_{X} c_{2}(X) L^{n-3} \\
& +1-h^{1}\left(\mathcal{O}_{X}\right)+h^{2}\left(\mathcal{O}_{X}\right) .
\end{aligned}
$$

THEOREM 2.1. Let $(X, L)$ be a polarized manifold with $\operatorname{dim} X=n$, and let $i$ be an integer with $0 \leq i \leq n-1$. Then

$$
g_{i}(X, L)=\sum_{j=0}^{n-i-1}(-1)^{j}\left(\begin{array}{c}
n-i \\
j
\end{array}\right) h^{0}\left(K_{X}+(n-i-j) L\right)+\sum_{k=0}^{n-i}(-1)^{n-i-k} h^{n-k}\left(\mathcal{O}_{X}\right) .
$$

Proof. See [9, Theorem 2.3].

Definition 2.2. [12, Definitions 3.1 and 3.2] Let $(X, L)$ be a polarized manifold of dimension $n$.

(i) Let $t$ be a positive integer. Then set

$$
\begin{aligned}
& F_{0}(t):=h^{0}\left(K_{X}+t L\right), \\
& F_{i}(t):=F_{i-1}(t+1)-F_{i-1}(t) \text { for every integer } i \text { satisfying } 1 \leq i \leq n .
\end{aligned}
$$

(ii) For every integer $i$ satisfying $0 \leq i \leq n$, the ith Hilbert coefficient, $A_{i}(X, L)$, of $(X, L)$ is defined by $A_{i}(X, L)=F_{n-i}(1)$.

\section{Remark 2.2.}

(i) If $1 \leq i \leq n$, then $A_{i}(X, L)$ can be defined as follows (see [12, Proposition 3.2]):

$$
A_{i}(X, L)=g_{i}(X, L)+g_{i-1}(X, L)-h^{i-1}\left(\mathcal{O}_{X}\right) .
$$

(ii) By employing Definition 2.2 and [12, Proposition 3.1(2)], we obtain the following:

(ii.1) $A_{i}(X, L) \in \mathbb{Z}$ for every integer $i$ satisfying $0 \leq i \leq n$;

(ii.2) $A_{0}(X, L)=L^{n}$;

(ii.3) $A_{1}(X, L)=g(X, L)+L^{n}-1 \geq 0$ (see Remark 2.1(iii) and (iv));

(ii.4) $A_{n}(X, L)=h^{0}\left(K_{X}+L\right)$.

(iii) By applying Remark 2.1(v) and (vi) and Remark 2.2(i), we observe that $A_{2}(X, L)$ and $A_{3}(X, L)$ are respectively given by

$$
\begin{aligned}
A_{2}(X, L)= & \frac{(3 n-2)(n+1)}{24} L^{n}+\frac{n}{4} K_{X} L^{n-1}+\frac{1}{12}\left(K_{X}^{2}+c_{2}(X)\right) L^{n-2}, \\
A_{3}(X, L)= & \frac{(n-2)\left(n^{2}-1\right)}{48} L^{n}+\frac{n(3 n-5)}{48} K_{X} L^{n-1}+\frac{n-1}{24} K_{X}^{2} L^{n-2} \\
& +\frac{1}{24} c_{2}(X)\left(K_{X}+(n-1) L\right) L^{n-3} .
\end{aligned}
$$


THEOREM 2.2. Let $(X, L)$ be a polarized manifold of dimension $n$ and let $t$ be a positive integer. Then, for every integer $i$ satisfying $0 \leq i \leq n$, we have

$$
F_{n-i}(t)=\sum_{j=0}^{i}\left(\begin{array}{l}
t-1 \\
i-j
\end{array}\right) A_{j}(X, L) .
$$

Proof. See [12, Theorem 3.1].

COROLlary 2.1. [12, Corollary 3.1] Let $(X, L)$ be a polarized manifold of dimension $n$, and let $t$ be a positive integer. Then, we have

$$
h^{0}\left(K_{X}+t L\right)=\sum_{j=0}^{n}\left(\begin{array}{l}
t-1 \\
n-j
\end{array}\right) A_{j}(X, L) .
$$

THEOREM 2.3. Let $X$ be a projective manifold. Then there exist smooth projective varieties $X^{\prime}$ and $Y$, a birational morphism $\mu: X^{\prime} \rightarrow X$ and a fiber space $\phi: X^{\prime} \rightarrow Y$ such that $Y$ is not uniruled, and if $\operatorname{dim} X^{\prime}>\operatorname{dim} Y$, then the general fiber of $\phi$ is rationally connected.

Proof. See [5], [17] and [21].

Definition 2.3. The fiber space $\phi: X^{\prime} \rightarrow Y$ in Theorem 2.3 is called the maximal rationally connected fibration (MRC-fibration) of $X$, while $Y$ is called the base of the MRC-fibration.

Proposition 2.1. Let $(X, A)$ be a polarized manifold of dimension $n$. Assume that $h^{0}\left(K_{X}+A\right)>0$. Then $\Omega_{X}\langle A\rangle$ is generically nef.

Proof. See [16, Claim 2.1].

PROPOSITION 2.2. Let $X$ be a normal projective variety, and let $L$ and $M$ be a line bundle on $X$ such that $h^{0}(L)>0$ and $h^{0}(M)>0$, respectively. Then, $h^{0}(L+M) \geq h^{0}(L)+h^{0}(M)-$ 1 holds.

Proof. See [20, 15.6.2 Lemma].

\section{Main result}

THEOREM 3.1. Let $(X, L)$ be a polarized manifold of dimension five such that $h^{0}(L)>0$. Then $h^{0}\left(K_{X}+m L\right) \geq\left(\begin{array}{c}m-1 \\ 5\end{array}\right)$ holds for every integer $m \geq 6$. If equality holds for some $m \geq 6$, then $(X, L) \cong\left(\mathbb{P}^{n}, \mathcal{O}_{\mathbb{P}^{n}}(1)\right)$.

Proof. Suppose that $K_{X}+4 L$ is not nef. Then $(X, L)$ can be one of the following three types since $n=5$ (see [7, Theorems (11.2) and (11.7)] and [2, Theorems 7.2.1, 7.2.3, 7.2.4 and Proposition 7.2.2]):

(a) $\quad\left(\mathbb{P}^{5}, \mathcal{O}_{\mathbb{P}^{5}}(1)\right)$;

(b) $\left(\mathbb{Q}^{5}, \mathcal{O}_{\mathbb{Q}^{5}}(1)\right)$;

(c) a scroll over a smooth projective curve.

In these cases, we observe from [16, Remark 2.4] that Theorem 3.1 holds. Therefore we can assume that $K_{X}+4 L$ is nef. 
First, we get from Corollary 2.1 that the following equality holds:

$$
h^{0}\left(K_{X}+m L\right)=\sum_{i=0}^{5}\left(\begin{array}{c}
m-1 \\
5-j
\end{array}\right) A_{j}(X, L) .
$$

Since $A_{5}(X, L)=h^{0}\left(K_{X}+L\right)$, we have

$$
A_{5}(X, L) \geq 0
$$

Claim 3.1. $A_{4}(X, L) \geq 0$.

Proof. We note that $A_{4}(X, L)=h^{0}\left(K_{X}+2 L\right)-h^{0}\left(K_{X}+L\right)$. If $h^{0}\left(K_{X}+L\right)=0$, then $A_{4}(X, L)=h^{0}\left(K_{X}+2 L\right) \geq 0$. Thus, we may assume that $h^{0}\left(K_{X}+L\right)>0$. It follows that $h^{0}\left(K_{X}+2 L\right) \geq h^{0}\left(K_{X}+L\right)+h^{0}(L)-1$ holds by utilizing Proposition 2.2 because $h^{0}(L)>0$. Hence, $A_{4}(X, L)=h^{0}\left(K_{X}+2 L\right)-h^{0}\left(K_{X}+L\right) \geq h^{0}(L)-1 \geq 0$. Hence, this completes the assertion of Claim 3.1.

Additionally, we also note the following.

Claim 3.2. $A_{1}(X, L) \geq 1$.

Proof. We observe from Remark 2.2(ii.3) that $A_{1}(X, L) \geq 0$. Assume that $A_{0}(X, L)=0$. Then, $g(X, L)=0$ and $L^{5}=1$. It follows that $(X, L) \cong\left(\mathbb{P}^{5}, \mathcal{O}_{\mathbb{P}^{5}}(1)\right)$. This is impossible because we assumed that $K_{X}+4 L$ is nef.

We split the proof of Theorem 3.1 into three cases (i)-(iii) and many sub-cases.

(i) We consider the case that $h^{0}\left(K_{X}+2 L\right)=0$.

PROPOSITION 3.1. If $h^{0}\left(K_{X}+2 L\right)=0$, then $h^{0}\left(K_{X}+m L\right)>\left(\begin{array}{c}m-1 \\ 5\end{array}\right)$ for every integer $m \geq 6$.

Proof. By employing equation (1), inequality (2), Claim 3.1 and Remark 2.2(ii.2), we have

$$
h^{0}\left(K_{X}+m L\right)>\left(\begin{array}{c}
m-1 \\
2
\end{array}\right) A_{3}(X, L)+\left(\begin{array}{c}
m-1 \\
3
\end{array}\right) A_{2}(X, L) .
$$

Thus, to prove Proposition 3.1, it suffices to show that

$$
\left(\begin{array}{c}
m-1 \\
2
\end{array}\right) A_{3}(X, L)+\left(\begin{array}{c}
m-1 \\
3
\end{array}\right) A_{2}(X, L) \geq 0 .
$$

In this case, $h^{0}\left(K_{X}+L\right)=0$ holds since $h^{0}(L)>0$. Therefore we have $A_{3}(X, L)=$ $h^{0}\left(K_{X}+3 L\right)-2 h^{0}\left(K_{X}+2 L\right)+h^{0}\left(K_{X}+L\right)=h^{0}\left(K_{X}+3 L\right) \geq 0$. Thus, $A_{2}(X, L) \geq 0$ implies that inequality (3) holds. Furthermore we prove that $A_{2}(X, L) \geq 0$ in this case.

Let $\phi: X^{\prime} \rightarrow Y$ be the MRC-fibration of $X$, and let $Y$ be the base of the MRC-fibration, where $X^{\prime}$ and $Y$ are smooth projective varieties such that $X^{\prime}$ is birational to $X$.

(i.1) Let us consider the case that $\operatorname{dim} Y \geq 3$. Then, by utilizing [18, Step 2 on p. 741], we have $A_{2}(X, L) \geq 0$ (see also [14, p. 350]).

(i.2) We consider the case that $\operatorname{dim} Y=0$. Then, $h^{i}\left(\mathcal{O}_{X}\right)=0$, for any $i \geq 1$. Thus, $g_{2}(X, L)=$ $h^{0}\left(K_{X}+3 L\right) \geq 0$. Besides, we also note that $g_{1}(X, L) \geq 0=h^{1}\left(\mathcal{O}_{X}\right)$. Hence $A_{2}(X, L)=$ $g_{2}(X, L)+g_{1}(X, L)-h^{1}\left(\mathcal{O}_{X}\right) \geq 0$. 
(i.3) Let us consider the case that $\operatorname{dim} Y=1$. Then $h^{1}\left(\mathcal{O}_{X}\right)=h^{1}\left(\mathcal{O}_{Y}\right)$ holds because $h^{1}\left(\mathcal{O}_{F}\right)=0$. By applying [8, Theorem 1.2.1], we have $g_{1}(X, L) \geq h^{1}\left(\mathcal{O}_{Y}\right)=h^{1}\left(\mathcal{O}_{X}\right)$. Moreover, we get $g_{2}(X, L)=h^{0}\left(K_{X}+3 L\right) \geq 0$ since $h^{i}\left(\mathcal{O}_{X}\right)=0$, for any $i \geq 2$. Consequently, $A_{2}(X, L)=g_{2}(X, L)+g_{1}(X, L)-h^{1}\left(\mathcal{O}_{X}\right) \geq 0$.

(i.4) Let us consider the case that $\operatorname{dim} Y=2$. Here we note that

$$
\begin{aligned}
& g_{1}(X, L)=1+\frac{1}{2}\left(K_{X}+4 L\right) L^{4}, \\
& g_{2}(X, L)=h^{0}\left(K_{X}+3 L\right)+h^{2}\left(\mathcal{O}_{X}\right) .
\end{aligned}
$$

By utilizing (4) and (5), we have

$$
\begin{aligned}
A_{2}(X, L) & =g_{2}(X, L)+g_{1}(X, L)-h^{1}\left(\mathcal{O}_{X}\right) \\
& =h^{0}\left(K_{X}+3 L\right)+h^{2}\left(\mathcal{O}_{X}\right)-h^{1}\left(\mathcal{O}_{X}\right)+1+\frac{1}{2}\left(K_{X}+4 L\right) L^{4} \\
& \geq h^{0}\left(K_{X}+3 L\right)+h^{2}\left(\mathcal{O}_{Y}\right)-h^{1}\left(\mathcal{O}_{X}\right)+1+\frac{1}{2}\left(K_{X}+4 L\right) L^{4} \\
& =h^{0}\left(K_{X}+3 L\right)+\chi\left(\mathcal{O}_{Y}\right)+\frac{1}{2}\left(K_{X}+4 L\right) L^{4} .
\end{aligned}
$$

Since $Y$ is not uniruled, we have $\kappa(Y) \geq 0$ and $\chi\left(\mathcal{O}_{Y}\right) \geq 0$. Hence we obtain that $A_{2}(X, L) \geq 0$.

It follows from the above argument that $A_{2}(X, L) \geq 0$ and inequality (3) holds for the case where $h^{0}\left(K_{X}+2 L\right)=0$. This completes the proof of Proposition 3.1.

(ii) We consider the case that $h^{0}\left(K_{X}+2 L\right)>0$ and $h^{0}\left(K_{X}+L\right)=0$ and prove the following proposition.

Proposition 3.2. If $h^{0}\left(K_{X}+2 L\right)>0$ and $h^{0}\left(K_{X}+L\right)=0$, then

$$
h^{0}\left(K_{X}+m L\right)>\left(\begin{array}{c}
m-1 \\
5
\end{array}\right) \text { for every integer } m \geq 6
$$

Proof. First we note that

$$
\begin{aligned}
& \left(\begin{array}{c}
m-1 \\
2
\end{array}\right) A_{3}(X, L)+\left(\begin{array}{c}
m-1 \\
3
\end{array}\right) A_{2}(X, L)+\left(\begin{array}{c}
m-1 \\
4
\end{array}\right) A_{1}(X, L) \\
& =\frac{(m-1)(m-2)}{6}\left(3 A_{3}(X, L)+(m-3) A_{2}(X, L)+\frac{(m-3)(m-4)}{4} A_{1}(X, L)\right) .
\end{aligned}
$$

To prove this proposition, it suffices to show that

$$
3 A_{3}(X, L)+(m-3) A_{2}(X, L)+\frac{(m-3)(m-4)}{4} A_{1}(X, L)>0 .
$$

Using Proposition 2.1, we observe from $h^{0}\left(K_{X}+2 L\right)>0$ that $\Omega_{X}\langle 2 L\rangle$ is generically nef. Additionally, we note that $K_{X}+10 L$ is nef by applying the adjunction theory. Hence, by employing [18, 2.11 Corollary] we have

$$
c_{2}(X) H_{1} H_{2} H_{3} \geq-\left(8 K_{X} L+40 L^{2}\right) H_{1} H_{2} H_{3}
$$


for every nef line bundles $H_{1}, H_{2}$ and $H_{3}$. Therefore, we get the following by utilizing inequality (7):

$$
\begin{aligned}
& 3 A_{3}(X, L)+(m-3) A_{2}(X, L)+\frac{(m-3)(m-4)}{4} A_{1}(X, L) \\
&=\frac{3 m^{2}-8 m+15}{4} L^{5}+\frac{m^{2}+3 m+7}{8} K_{X} L^{4}+\frac{m+3}{12} K_{X}^{2} L^{3} \\
& \quad+\frac{3 K_{X}+(2 m+6) L}{24} c_{2}(X) L^{2} \\
& \geq \frac{m-9}{12} K_{X}^{2} L^{3}+\frac{3 m^{2}-7 m-147}{24} K_{X} L^{4}+\frac{18 m^{2}-128 m-150}{24} L^{5} \\
&=\frac{m-9}{24}\left(2 K_{X}^{2} L^{3}+(3 m+20) K_{X} L^{4}+(18 m+34) L^{5}\right)+\frac{1}{24}\left(33 K_{X}+156 L\right) L^{4} \\
&= \frac{m-9}{24}\left(\left(2 K_{X}+(3 m+16) L\right)\left(K_{X}+2 L\right) L^{3}+(12 m+2) L^{5}\right) \\
&+\frac{1}{24}\left(33 K_{X}+156 L\right) L^{4} .
\end{aligned}
$$

(ii.1) If $m \geq 9$, then inequality (6) holds by utilizing (8) because $K_{X}+4 L$ is nef.

(ii.2) Assume that $m=6$. Then, since $h^{0}(L)>0$ and [18, 1.2 Theorem], we have $h^{0}\left(K_{X}+\right.$ $6 L) \geq 1=\left(\begin{array}{c}6-1 \\ 5\end{array}\right)$. If $h^{0}\left(K_{X}+6 L\right)=1$, then $h^{0}\left(K_{X}+m L\right)=1$, for $m=4,5,6$, using $h^{0}(L)>0$ and $[18,1.2$ Theorem]. Thus, by applying Corollary 2.1 , we have

$$
\left.\begin{array}{l}
A_{5}(X, L)+3 A_{4}(X, L)+3 A_{3}(X, L)+A_{2}(X, L)=1 \\
A_{5}(X, L)+4 A_{4}(X, L)+6 A_{3}(X, L)+4 A_{2}(X, L)+A_{1}(X, L)=1, \\
A_{5}(X, L)+5 A_{4}(X, L)+10 A_{3}(X, L)+10 A_{2}(X, L)+5 A_{1}(X, L)+A_{0}(X, L)=1 .
\end{array}\right\}
$$

Since $A_{5}(X, L)=h^{0}\left(K_{X}+L\right)=0$, we observe from (9) that $5 A_{4}(X, L)=3-3 A_{0}(X, L)$ $-5 A_{1}(X, L) \leq-5 A_{1}(X, L) \leq-15$, which implies that $A_{4}(X, L) \leq-3$. However, this is impossible because $0<h^{0}\left(K_{X}+2 L\right)=A_{5}(X, L)+A_{4}(X, L)=A_{4}(X, L)$. Therefore, we obtain that $h^{0}\left(K_{X}+6 L\right)>1=\left(\begin{array}{c}6-1 \\ 5\end{array}\right)$.

(ii.3) We consider the case that $m=7$. First we prove the following claim.

ClaIm 3.3. In this case, we may assume that $A_{1}(X, L) \geq 3$.

Proof. Using Claim 3.2 we have $A_{1}(X, L) \geq 1$. Assume that $A_{1}(X, L)=1$. Then, since $A_{1}(X, L)=g(X, L)+L^{5}-1$, we have $g(X, L) \leq 1$. Moreover, by the classification of $(X, L)$ with $g(X, L) \leq 1$ (see [7, Theorems (12.1) and (12.3)]), we observe that $h^{0}\left(K_{X}+\right.$ $2 L)=0$ in this case. This contradicts the assumption.

Assume that $A_{1}(X, L)=2$. Then we have $2=A_{1}(X, L)=g(X, L)+L^{5}-1$. Since $L^{5} \geq 1$, we get $g(X, L) \leq 2$. If $g(X, L) \leq 1$, then $\left(K_{X}+4 L\right) L^{4} \leq 0$. However, this is impossible because $h^{0}\left(K_{X}+2 L\right)>0$ and $L$ is ample. Therefore $g(X, L)=2$ and $L^{5}=1$. Consequently, we observe that $\left(K_{X}+2 L\right) L^{4}=0$ because $g(X, L)=1+\frac{1}{2}\left(K_{X}+4 L\right) L^{4}$. Since $h^{0}\left(K_{X}+2 L\right)>0$ and $L$ is ample, we have $K_{X}+2 L=\mathcal{O}_{X}$ and $h^{0}\left(K_{X}+2 L\right)=1$. 
Thus, we obtain the following by applying Remark 2.2(ii.3):

$$
\begin{gathered}
A_{5}(X, L)=h^{0}\left(K_{X}+L\right)=0, \\
A_{4}(X, L)=h^{0}\left(K_{X}+2 L\right)-h^{0}\left(K_{X}+L\right)=1 .
\end{gathered}
$$

Since

$$
0 \leq h^{0}\left(K_{X}+3 L\right)-h^{0}\left(K_{X}+2 L\right)=A_{4}(X, L)+A_{3}(X, L)=1+A_{3}(X, L),
$$

we have

$$
A_{3}(X, L) \geq-1 \text {. }
$$

Next, we calculate $A_{2}(X, L)$. First, we calculate $g_{2}(X, L)$. Here, we note that $h^{i}\left(\mathcal{O}_{X}\right)=$ 0 , for every integer $i \geq 1$ since $K_{X}=-2 L$. Then, by applying Theorem 2.1, we have

$$
\begin{aligned}
g_{2}(X, L) & =h^{0}\left(K_{X}+3 L\right)-3 h^{0}\left(K_{X}+2 L\right)+3 h^{0}\left(K_{X}+L\right)-\sum_{k=0}^{3}(-1)^{3-k} h^{5-k}\left(\mathcal{O}_{X}\right) \\
& =h^{0}\left(K_{X}+3 L\right)-3 .
\end{aligned}
$$

Since $h^{0}(L)>0$ and $h^{0}\left(K_{X}+2 L\right)=1$, we have $h^{0}\left(K_{X}+3 L\right) \geq 1$. Hence, $g_{2}(X, L) \geq-2$. Thus, we get

$$
A_{2}(X, L)=g_{2}(X, L)+g_{1}(X, L)-h^{1}\left(\mathcal{O}_{X}\right) \geq 0 .
$$

Since $A_{1}(X, L)=2$ and $A_{0}(X, L)=L^{5}=1$, we observe from equation (1), equation (11), inequality (12) and inequality (13) that

$$
\begin{aligned}
h^{0}\left(K_{X}+7 L\right)= & A_{5}(X, L)+6 A_{4}(X, L)+15 A_{3}(X, L)+20 A_{2}(X, L)+15 A_{1}(X, L) \\
& +6 A_{0}(X, L) \\
\geq & 0+6-15+0+30+6 \\
= & 27>\left(\begin{array}{l}
6 \\
5
\end{array}\right) .
\end{aligned}
$$

Therefore we get the assertion of Claim 3.3.

If $h^{0}\left(K_{X}+p L\right) \geq 7$, for some integer $p$ that satisfies $1 \leq p \leq 6$, then $h^{0}\left(K_{X}+7 L\right) \geq$ $h^{0}\left(K_{X}+6 L\right)+h^{0}(L)-1 \geq 7>\left(\begin{array}{c}7-1 \\ 5\end{array}\right)$. Thus, we may assume that

$$
h^{0}\left(K_{X}+p L\right) \leq 6 \text { for any integer } p \text { with } 1 \leq p \leq 6 .
$$

Hence, we obtain the following using equation (10):

$$
\begin{aligned}
6 & \geq h^{0}\left(K_{X}+3 L\right) \\
& =A_{5}(X, L)+2 A_{4}(X, L)+A_{3}(X, L)=2 A_{4}(X, L)+A_{3}(X, L) .
\end{aligned}
$$

Therefore,

$$
A_{3}(X, L) \leq 6-2 A_{4}(X, L)
$$

Now, we note that

$$
0 \leq h^{0}(L)-1 \leq h^{0}\left(K_{X}+3 L\right)-h^{0}\left(K_{X}+2 L\right)=A_{4}(X, L)+A_{3}(X, L) .
$$


Moreover, we get

$$
\begin{aligned}
1 \leq h^{0}\left(K_{X}+4 L\right) & =A_{5}(X, L)+3 A_{4}(X, L)+3 A_{3}(X, L)+A_{2}(X, L) \\
& =3 A_{4}(X, L)+3 A_{3}(X, L)+A_{2}(X, L) .
\end{aligned}
$$

Hence, inequality (15) gives

$$
\begin{aligned}
A_{2}(X, L) & \geq-3 A_{4}(X, L)-3 A_{3}(X, L)+1 \\
& \geq-3 A_{4}(X, L)+3\left(2 A_{4}(X, L)-6\right)+1 \\
& =3 A_{4}(X, L)-17
\end{aligned}
$$

We note that the following hold by employing inequality (14):

$$
1 \leq h^{0}\left(K_{X}+2 L\right)=A_{5}(X, L)+A_{4}(X, L) \leq 6 .
$$

Thus, we observe from equation (10) that

$$
1 \leq A_{4}(X, L) \leq 6
$$

(ii.3.1) Let us consider the case that $A_{4}(X, L)=6$. Since $A_{4}(X, L)+A_{3}(X, L) \geq 0$ by applying inequality (16), we have $A_{3}(X, L) \geq-6$. Moreover, inequality (17) gives that $A_{2}(X, L) \geq 1$. Hence, using Claim 3.3, we observe that

$$
\begin{aligned}
h^{0}\left(K_{X}+7 L\right)= & A_{5}(X, L)+6 A_{4}(X, L)+15 A_{3}(X, L) \\
& +20 A_{2}(X, L)+15 A_{1}(X, L)+6 A_{0}(X, L) \\
\geq & 36-90+20+45+6=17 .
\end{aligned}
$$

Hence the assertion holds.

(ii.3.2) We consider the case that $A_{4}(X, L)=5$. Since $A_{4}(X, L)+A_{3}(X, L) \geq 0$ by employing inequality (16), we have $A_{3}(X, L) \geq-5$. However, $A_{3}(X, L) \leq-4$ by utilizing inequality (15). Hence $A_{3}(X, L)=-5$ or -4 . Moreover, using inequality (17), we obtain $A_{2}(X, L) \geq-2$.

It follows that

$$
\begin{aligned}
h^{0}\left(K_{X}+7 L\right)= & A_{5}(X, L)+6 A_{4}(X, L)+15 A_{3}(X, L) \\
& +20 A_{2}(X, L)+15 A_{1}(X, L)+6 A_{0}(X, L) \\
\geq & 30-75+20 A_{2}(X, L)+45+6 \\
= & 6+20 A_{2}(X, L) .
\end{aligned}
$$

Hence, if $A_{2}(X, L) \geq 1$, then we obtain the assertion. Thus, we may assume that $A_{2}(X, L) \leq 0$. Inequality (17) gives that $A_{2}(X, L) \geq-2$. Therefore, we have $A_{2}(X, L)=$ 
$0,-1$ or -2 . Furthermore

$$
\begin{aligned}
1 & \leq h^{0}\left(K_{X}+5 L\right) \\
= & A_{5}(X, L)+4 A_{4}(X, L)+6 A_{3}(X, L)+4 A_{2}(X, L)+A_{1}(X, L) \\
& = \begin{cases}-18+A_{1}(X, L), & \text { if }\left(A_{3}(X, L), A_{2}(X, L)\right)=(-5,-2), \\
-14+A_{1}(X, L), & \text { if }\left(A_{3}(X, L), A_{2}(X, L)\right)=(-5,-1), \\
-10+A_{1}(X, L), & \text { if }\left(A_{3}(X, L), A_{2}(X, L)\right)=(-5,0), \\
-12+A_{1}(X, L), & \text { if }\left(A_{3}(X, L), A_{2}(X, L)\right)=(-4,-2), \\
-8+A_{1}(X, L), & \text { if }\left(A_{3}(X, L), A_{2}(X, L)\right)=(-4,-1), \\
-4+A_{1}(X, L), & \text { if }\left(A_{3}(X, L), A_{2}(X, L)\right)=(-4,0),\end{cases}
\end{aligned}
$$

Consequently, we observe that

$$
A_{1}(X, L) \geq \begin{cases}19, & \text { if }\left(A_{3}(X, L), A_{2}(X, L)\right)=(-5,-2), \\ 15, & \text { if }\left(A_{3}(X, L), A_{2}(X, L)\right)=(-5,-1), \\ 11, & \text { if }\left(A_{3}(X, L), A_{2}(X, L)\right)=(-5,0), \\ 13, & \text { if }\left(A_{3}(X, L), A_{2}(X, L)\right)=(-4,-2), \\ 9, & \text { if }\left(A_{3}(X, L), A_{2}(X, L)\right)=(-4,-1), \\ 5, & \text { if }\left(A_{3}(X, L), A_{2}(X, L)\right)=(-4,0) .\end{cases}
$$

Hence, we get the assertion for the case that $A_{4}(X, L)=5$.

(ii.3.3) We consider the case that $A_{4}(X, L)=4$. In this case, we get $A_{3}(X, L) \geq-4$ by applying inequality (16). However, we obtain $A_{3}(X, L) \leq-2$ by employing inequality (15). Thus, we have $\left(A_{4}(X, L), A_{3}(X, L)\right)=(4,-4),(4,-3)$ or $(4,-2)$.

(ii.3.3.1) We now consider the case that $\left(A_{4}(X, L), A_{3}(X, L)\right)=(4,-4)$. Then, we note that

$$
A_{2}(X, L) \geq-3 A_{4}(X, L)-3 A_{3}(X, L)+1=1
$$

using inequality (17). Thus, we obtain that

$$
\begin{aligned}
h^{0} & \left(K_{X}+7 L\right) \\
& =A_{5}(X, L)+6 A_{4}(X, L)+15 A_{3}(X, L)+20 A_{2}(X, L)+15 A_{1}(X, L)+6 A_{0}(X, L) \\
& \geq 35 .
\end{aligned}
$$

Thus, we get the assertion.

(ii.3.3.2) We consider the case that $\left(A_{4}(X, L), A_{3}(X, L)\right)=(4,-3)$. Then we note that

$$
A_{2}(X, L) \geq-3 A_{4}(X, L)-3 A_{3}(X, L)+1=-2
$$

by applying inequality (17). Here we assume that $7>h^{0}\left(K_{X}+7 L\right)$. Then, using equation (10), Claim 3.3 and assumptions, we have

$$
\begin{aligned}
7 & >h^{0}\left(K_{X}+7 L\right) \\
& =A_{5}(X, L)+6 A_{4}(X, L)+15 A_{3}(X, L)+20 A_{2}(X, L)+15 A_{1}(X, L)+6 A_{0}(X, L) \\
& \geq 20 A_{2}(X, L)+30 .
\end{aligned}
$$


Thus, we get

$$
A_{2}(X, L) \leq-1 .
$$

By utilizing inequalities (18) and (19), we have $A_{2}(X, L)=-1$ or -2 . Therefore, it follows that

$$
\begin{aligned}
1 & \leq h^{0}\left(K_{X}+5 L\right) \\
& =A_{5}(X, L)+4 A_{4}(X, L)+6 A_{3}(X, L)+4 A_{2}(X, L)+A_{1}(X, L) \\
& = \begin{cases}-6+A_{1}(X, L), & \text { if } A_{2}(X, L)=-1, \\
-10+A_{1}(X, L), & \text { if } A_{2}(X, L)=-2 .\end{cases}
\end{aligned}
$$

Thus, we have

$$
A_{1}(X, L) \geq \begin{cases}7, & \text { if } A_{2}(X, L)=-1 \\ 11, & \text { if } A_{2}(X, L)=-2\end{cases}
$$

Consequently, we obtain that

$$
\begin{aligned}
h^{0}\left(K_{X}+7 L\right)= & A_{5}(X, L)+6 A_{4}(X, L)+15 A_{3}(X, L) \\
& +20 A_{2}(X, L)+15 A_{1}(X, L)+6 A_{0}(X, L) \\
\geq & \begin{cases}24-45-20+105+6>7, & \text { if } A_{2}(X, L)=-1, \\
24-45-40+105+6>7, & \text { if } A_{2}(X, L)=-2 .\end{cases}
\end{aligned}
$$

This contradicts the assumption that $h^{0}\left(K_{X}+7 L\right)<7$.

(ii.3.3.3) We consider the case that $\left(A_{4}(X, L), A_{3}(X, L)\right)=(4,-2)$. Then, we note that

$$
A_{2}(X, L) \geq-3 A_{4}(X, L)-3 A_{3}(X, L)+1=-5
$$

by employing inequality (17). Here, we assume that $7>h^{0}\left(K_{X}+7 L\right)$. Then, using equation (10), Claim 3.3 and assumptions, we obtain that

$$
\begin{aligned}
7 & >h^{0}\left(K_{X}+7 L\right) \\
& =A_{5}(X, L)+6 A_{4}(X, L)+15 A_{3}(X, L)+20 A_{2}(X, L)+15 A_{1}(X, L)+6 A_{0}(X, L) \\
& \geq 20 A_{2}(X, L)+45 .
\end{aligned}
$$

Therefore we get

$$
A_{2}(X, L) \leq-1 .
$$

By applying inequalities (20) and (21), we have $-5 \leq A_{2}(X, L) \leq-1$.

(ii.3.3.3.1) Assume that $A_{2}(X, L)=-1$. Then, using equation (10), Claim 3.3 and assumptions, we obtain that

$$
\begin{aligned}
h^{0}\left(K_{X}+7 L\right)= & A_{5}(X, L)+6 A_{4}(X, L)+15 A_{3}(X, L) \\
& +20 A_{2}(X, L)+15 A_{1}(X, L)+6 A_{0}(X, L) \\
\geq & 24-30-20+45+6>6 .
\end{aligned}
$$

This contradicts the assumption that $h^{0}\left(K_{X}+7 L\right)<7$. 
(ii.3.3.3.2) Assume that $A_{2}(X, L)=-2$. Then, it is not difficult to see that

$$
\begin{aligned}
1 & \leq h^{0}\left(K_{X}+5 L\right) \\
& =A_{5}(X, L)+4 A_{4}(X, L)+6 A_{3}(X, L)+4 A_{2}(X, L)+A_{1}(X, L) \\
& =-4+A_{1}(X, L) .
\end{aligned}
$$

Thus, we get $A_{1}(X, L) \geq 5$. Therefore, it follows that

$$
\begin{aligned}
h^{0}\left(K_{X}+7 L\right)= & A_{5}(X, L)+6 A_{4}(X, L)+15 A_{3}(X, L) \\
& +20 A_{2}(X, L)+15 A_{1}(X, L)+6 A_{0}(X, L) \\
\geq & 24-30-40+75+6>6 .
\end{aligned}
$$

This also contradicts the assumption that $h^{0}\left(K_{X}+7 L\right) \leq 7$.

(ii.3.3.3.3) Assume that $-5 \leq A_{2}(X, L) \leq-3$. Since $A_{2}(X, L) \leq-3$, one can easily see that

$$
\begin{aligned}
1 & \leq h^{0}\left(K_{X}+5 L\right) \\
& =A_{5}(X, L)+4 A_{4}(X, L)+6 A_{3}(X, L)+4 A_{2}(X, L)+A_{1}(X, L) \\
& \leq-8+A_{1}(X, L) .
\end{aligned}
$$

Thus, we have $A_{1}(X, L) \geq 9$. Therefore, it follows that

$$
\begin{aligned}
h^{0}\left(K_{X}+7 L\right)= & A_{5}(X, L)+6 A_{4}(X, L)+15 A_{3}(X, L) \\
& +20 A_{2}(X, L)+15 A_{1}(X, L)+6 A_{0}(X, L) \\
\geq & 24-30-100+135+6>6 .
\end{aligned}
$$

This also contradicts the assumption that $h^{0}\left(K_{X}+7 L\right)<7$.

(ii.3.4) Let us consider the case that $A_{4}(X, L)=3$. This case yields that $A_{3}(X, L) \geq-3$ by utilizing inequality (16). However, we have $A_{3}(X, L) \leq 0$ by applying inequality (15). Hence, we have $\left(A_{4}(X, L), A_{3}(X, L)\right)=(3,-3),(3,-2),(3,-1)$ or $(3,0)$.

(ii.3.4.1) We consider the case that $\left(A_{4}(X, L), A_{3}(X, L)\right)=(3,-3)$. Then, we note that

$$
A_{2}(X, L) \geq-3 A_{4}(X, L)-3 A_{3}(X, L)+1=1
$$

by utilizing inequality (17). Thus, we observe that

$$
\begin{aligned}
& h^{0}\left(K_{X}+7 L\right) \\
& \quad \geq A_{5}(X, L)+6 A_{4}(X, L)+15 A_{3}(X, L)+20 A_{2}(X, L)+15 A_{1}(X, L)+6 A_{0}(X, L) \\
& \quad \geq 44 .
\end{aligned}
$$

Consequently, we obtain the assertion.

(ii.3.4.2) Let us consider the case that $\left(A_{4}(X, L), A_{3}(X, L)\right)=(3,-2)$. Then, using inequality (17) we obtain that

$$
A_{2}(X, L) \geq-3 A_{4}(X, L)-3 A_{3}(X, L)+1=-2 .
$$


Here, we assume that $7>h^{0}\left(K_{X}+7 L\right)$. Then, by applying equation (10), Claim 3.3 and assumptions, it follows that

$$
\begin{aligned}
7 & >h^{0}\left(K_{X}+7 L\right) \\
& =A_{5}(X, L)+6 A_{4}(X, L)+15 A_{3}(X, L)+20 A_{2}(X, L)+15 A_{1}(X, L)+6 A_{0}(X, L) \\
& \geq 20 A_{2}(X, L)+39 .
\end{aligned}
$$

This implies that

$$
A_{2}(X, L) \leq-1
$$

By employing inequalities (23) and (24), we have $A_{2}(X, L)=-1$ or -2 . Then, we get

$$
\begin{aligned}
1 & \leq h^{0}\left(K_{X}+5 L\right) \\
& =A_{5}(X, L)+4 A_{4}(X, L)+6 A_{3}(X, L)+4 A_{2}(X, L)+A_{1}(X, L) \\
& = \begin{cases}-4+A_{1}(X, L), & \text { if } A_{2}(X, L)=-1, \\
-8+A_{1}(X, L), & \text { if } A_{2}(X, L)=-2 .\end{cases}
\end{aligned}
$$

This yields that $A_{1}(X, L) \geq 5$. Therefore, we have

$$
\begin{aligned}
h^{0}\left(K_{X}+7 L\right)= & A_{5}(X, L)+6 A_{4}(X, L)+15 A_{3}(X, L) \\
& +20 A_{2}(X, L)+15 A_{1}(X, L)+6 A_{0}(X, L) \\
\geq & \begin{cases}18-30-20+75+6>7, & \text { if } A_{2}(X, L)=-1, \\
18-30-40+135+6>7, & \text { if } A_{2}(X, L)=-2 .\end{cases}
\end{aligned}
$$

This contradicts the assumption that $h^{0}\left(K_{X}+7 L\right)<7$.

(ii.3.4.3) We consider the case that $\left(A_{4}(X, L), A_{3}(X, L)\right)=(3,-1)$. Then, we observe that

$$
A_{2}(X, L) \geq-3 A_{4}(X, L)-3 A_{3}(X, L)+1=-5
$$

by applying inequality (17). Here, we assume that $7>h^{0}\left(K_{X}+7 L\right)$. Then, using equation (10), Claim 3.3 and assumptions, we have

$$
\begin{aligned}
7 & >h^{0}\left(K_{X}+7 L\right) \\
& =A_{5}(X, L)+6 A_{4}(X, L)+15 A_{3}(X, L)+20 A_{2}(X, L)+15 A_{1}(X, L)+6 A_{0}(X, L) \\
& \geq 20 A_{2}(X, L)+54 .
\end{aligned}
$$

It follows that

$$
A_{2}(X, L) \leq-2 .
$$

By using inequalities (25) and (26), we observe that $-5 \leq A_{2}(X, L) \leq-2$.

(ii.3.4.3.1) Assume that $-2 \leq A_{2}(X, L) \leq-1$. Then, by employing equation (10), Claim 3.3 and assumptions, we have

$$
\begin{aligned}
h^{0}\left(K_{X}+7 L\right)= & A_{5}(X, L)+6 A_{4}(X, L)+15 A_{3}(X, L) \\
& +20 A_{2}(X, L)+15 A_{1}(X, L)+6 A_{0}(X, L) \\
\geq & 18-15-40+45+6>7 .
\end{aligned}
$$


(ii.3.4.3.2) Assume that $A_{2}(X, L)=-3$ (respectively -4 or -5 ). Then, we get

$$
\begin{aligned}
1 & \leq h^{0}\left(K_{X}+5 L\right) \\
& =A_{5}(X, L)+4 A_{4}(X, L)+6 A_{3}(X, L)+4 A_{2}(X, L)+A_{1}(X, L) \\
& = \begin{cases}-6+A_{1}(X, L), & \text { if } A_{2}(X, L)=-3, \\
-10+A_{1}(X, L), & \text { if } A_{2}(X, L)=-4, \\
-14+A_{1}(X, L), & \text { if } A_{2}(X, L)=-5 .\end{cases}
\end{aligned}
$$

Thus, we have

$$
A_{1}(X, L) \geq \begin{cases}7, & \text { if } A_{2}(X, L)=-3 \\ 11, & \text { if } A_{2}(X, L)=-4 \\ 15, & \text { if } A_{2}(X, L)=-5\end{cases}
$$

Therefore, it follows that

$$
\begin{aligned}
h^{0}\left(K_{X}+7 L\right)= & A_{5}(X, L)+6 A_{4}(X, L)+15 A_{3}(X, L) \\
& +20 A_{2}(X, L)+15 A_{1}(X, L)+6 A_{0}(X, L) \\
> & 6 .
\end{aligned}
$$

This also contradicts the assumption that $h^{0}\left(K_{X}+7 L\right)<7$.

(ii.3.4.4) Let us consider the case that $\left(A_{4}(X, L), A_{3}(X, L)\right)=(3,0)$. Then, we observe that

$$
A_{2}(X, L) \geq-3 A_{4}(X, L)-3 A_{3}(X, L)+1=-8
$$

by utilizing inequality (17). Here, we assume that $7>h^{0}\left(K_{X}+7 L\right)$. Then, using (10), Claim 3.3 and assumptions, it follows that

$$
\begin{aligned}
7 & >h^{0}\left(K_{X}+7 L\right) \\
& =A_{5}(X, L)+6 A_{4}(X, L)+15 A_{3}(X, L)+20 A_{2}(X, L)+15 A_{1}(X, L)+6 A_{0}(X, L) \\
& \geq 20 A_{2}(X, L)+69 .
\end{aligned}
$$

Thus, we get

$$
A_{2}(X, L) \leq-3 .
$$

By employing inequalities (27) and (28), we have $-8 \leq A_{2}(X, L) \leq-3$.

(ii.3.4.4.1) Assume that $A_{2}(X, L)=-4$ (respectively $-5,-6,-7$ or -8 ). Then, it follows that

$$
\begin{aligned}
1 & \leq h^{0}\left(K_{X}+5 L\right) \\
= & A_{5}(X, L)+4 A_{4}(X, L)+6 A_{3}(X, L)+4 A_{2}(X, L)+A_{1}(X, L) \\
& = \begin{cases}-4+A_{1}(X, L), & \text { if } A_{2}(X, L)=-4, \\
-8+A_{1}(X, L), & \text { if } A_{2}(X, L)=-5, \\
-12+A_{1}(X, L), & \text { if } A_{2}(X, L)=-6, \\
-16+A_{1}(X, L), & \text { if } A_{2}(X, L)=-7, \\
-20+A_{1}(X, L), & \text { if } A_{2}(X, L)=-8 .\end{cases}
\end{aligned}
$$


Thus, we have

$$
A_{1}(X, L) \geq \begin{cases}5, & \text { if } A_{2}(X, L)=-4, \\ 9, & \text { if } A_{2}(X, L)=-5, \\ 13, & \text { if } A_{2}(X, L)=-6, \\ 17, & \text { if } A_{2}(X, L)=-7, \\ 21, & \text { if } A_{2}(X, L)=-8 .\end{cases}
$$

Therefore, one can see that

$$
\begin{aligned}
h^{0}\left(K_{X}+7 L\right)= & A_{5}(X, L)+6 A_{4}(X, L)+15 A_{3}(X, L) \\
& +20 A_{2}(X, L)+15 A_{1}(X, L)+6 A_{0}(X, L) \\
> & 6 .
\end{aligned}
$$

This also contradicts the assumption that $h^{0}\left(K_{X}+7 L\right)<7$.

(ii.3.4.4.2) Assume that $A_{2}(X, L)=-3$. Then, using equation (10), Claim 3.3 and assumptions, we observe that

$$
\begin{aligned}
h^{0}\left(K_{X}+7 L\right)= & A_{5}(X, L)+6 A_{4}(X, L)+15 A_{3}(X, L) \\
& +20 A_{2}(X, L)+15 A_{1}(X, L)+6 A_{0}(X, L) \\
\geq & 18-60+45+6>7 .
\end{aligned}
$$

(ii.3.5) We consider the case that $A_{4}(X, L)=2$. This case yields that $A_{3}(X, L) \geq-2$ by utilizing inequality (16). However, we get $A_{3}(X, L) \leq 2$ by applying inequality (15). Thus, we have

$$
\left(A_{4}(X, L), A_{3}(X, L)\right)=(2,-2),(2,-1),(2,0),(2,1) \text { or }(2,2) .
$$

(ii.3.5.1) We consider the case that $\left(A_{4}(X, L), A_{3}(X, L)\right)=(2,-2)$ or $(2,-1)$. Then, we observe that

$$
A_{2}(X, L) \geq-3 A_{4}(X, L)-3 A_{3}(X, L)+1= \begin{cases}1, & \text { if } A_{3}(X, L)=-2, \\ -2, & \text { if } A_{3}(X, L)=-1\end{cases}
$$

by employing inequality (17). Thus, we have

$$
\begin{aligned}
& h^{0}\left(K_{X}+7 L\right) \\
& \quad \geq A_{5}(X, L)+6 A_{4}(X, L)+15 A_{3}(X, L)+20 A_{2}(X, L)+15 A_{1}(X, L)+6 A_{0}(X, L) \\
& \quad \geq \begin{cases}44, & \text { if } A_{3}(X, L)=-2, \\
8, & \text { if } A_{3}(X, L)=-1 .\end{cases}
\end{aligned}
$$

Hence, we get the assertion. 
(ii.3.5.2) Let us consider the case that $\left(A_{4}(X, L), A_{3}(X, L)\right)=(2,0),(2,1)$ or $(2,2)$. Then we note that

$$
\begin{aligned}
h^{0}\left(K_{X}+3 L\right) & =A_{5}(X, L)+2 A_{4}(X, L)+A_{3}(X, L) \\
& =2 A_{4}(X, L)+3 A_{3}(X, L) \\
& = \begin{cases}4, & \text { if } A_{3}(X, L)=0, \\
5, & \text { if } A_{3}(X, L)=1, \\
6, & \text { if } A_{3}(X, L)=2 .\end{cases}
\end{aligned}
$$

Since $h^{0}(L)>0$, it follows from equation (29) that

$$
h^{0}\left(K_{X}+4 L\right) \geq \begin{cases}4, & \text { if } A_{3}(X, L)=0 \\ 5, & \text { if } A_{3}(X, L)=1 \\ 6, & \text { if } A_{3}(X, L)=2 .\end{cases}
$$

Since

$$
h^{0}\left(K_{X}+4 L\right)=A_{5}(X, L)+3 A_{4}(X, L)+3 A_{3}(X, L)+A_{2}(X, L),
$$

inequality (30) gives that

$$
A_{2}(X, L) \geq \begin{cases}-2, & \text { if } A_{3}(X, L)=0 \\ -4, & \text { if } A_{3}(X, L)=1 \\ -6, & \text { if } A_{3}(X, L)=2\end{cases}
$$

Here, we assume that $7>h^{0}\left(K_{X}+7 L\right)$. Then, by employing equation (10), Claim 3.3 and assumptions, we observe that

$$
\begin{aligned}
7 & h^{0}\left(K_{X}+7 L\right) \\
& =A_{5}(X, L)+6 A_{4}(X, L)+15 A_{3}(X, L)+20 A_{2}(X, L)+15 A_{1}(X, L)+6 A_{0}(X, L) \\
& \geq \begin{cases}20 A_{2}(X, L)+63, & \text { if } A_{3}(X, L)=0, \\
20 A_{2}(X, L)+78, & \text { if } A_{3}(X, L)=1, \\
20 A_{2}(X, L)+93, & \text { if } A_{3}(X, L)=2 .\end{cases}
\end{aligned}
$$

It follows that

$$
A_{2}(X, L) \leq \begin{cases}-2, & \text { if } A_{3}(X, L)=0 \\ -4, & \text { if } A_{3}(X, L)=1 \\ -5, & \text { if } A_{3}(X, L)=2 .\end{cases}
$$

(ii.3.5.2.1) Assume that $\left(A_{4}(X, L), A_{3}(X, L), A_{2}(X, L)\right)=(2,0,-2) \quad$ (respectively $(2,1,-4))$. Since $h^{0}(L)>0$, equation (29) gives that

$$
h^{0}\left(K_{X}+5 L\right) \geq \begin{cases}4, & \text { if }\left(A_{3}(X, L), A_{2}(X, L)\right)=(0,-2), \\ 5, & \text { if }\left(A_{3}(X, L), A_{2}(X, L)\right)=(1,-4) .\end{cases}
$$


Meanwhile, we observe that

$$
\begin{aligned}
h^{0}\left(K_{X}+5 L\right)= & A_{5}(X, L)+4 A_{4}(X, L)+6 A_{3}(X, L) \\
& +4 A_{2}(X, L)+A_{1}(X, L) .
\end{aligned}
$$

Therefore, we observe from inequality (31) and (32) that

$$
A_{1}(X, L) \geq \begin{cases}4, & \text { if }\left(A_{3}(X, L), A_{2}(X, L)\right)=(0,-2), \\ 7, & \text { if }\left(A_{3}(X, L), A_{2}(X, L)\right)=(1,-4)\end{cases}
$$

Therefore, it follows that

$$
\begin{aligned}
h^{0}\left(K_{X}+7 L\right)= & A_{5}(X, L)+6 A_{4}(X, L)+15 A_{3}(X, L) \\
& +20 A_{2}(X, L)+15 A_{1}(X, L)+6 A_{0}(X, L) \\
> & 7 .
\end{aligned}
$$

This contradicts the assumption that $h^{0}\left(K_{X}+7 L\right)<7$.

(ii.3.5.2.2) Assume that $\left(A_{4}(X, L), A_{3}(X, L), A_{2}(X, L)\right)=(2,2,-5) \quad$ (respectively $(2,2,-6))$. Since $h^{0}(L)>0$, equation (29) gives that

$$
h^{0}\left(K_{X}+5 L\right) \geq 6 .
$$

Hence, we see from inequality (33) and (32) that

$$
A_{1}(X, L) \geq \begin{cases}6, & \text { if } A_{2}(X, L)=-5 \\ 10, & \text { if } A_{2}(X, L)=-6\end{cases}
$$

Therefore, we get

$$
\begin{aligned}
h^{0}\left(K_{X}+7 L\right)= & A_{5}(X, L)+6 A_{4}(X, L)+15 A_{3}(X, L) \\
& +20 A_{2}(X, L)+15 A_{1}(X, L)+6 A_{0}(X, L) \\
> & 7 .
\end{aligned}
$$

This contradicts the assumption that $h^{0}\left(K_{X}+7 L\right)<7$.

(ii.3.6) We consider the case that $A_{4}(X, L)=1$. Using inequality (16), this case yields that $A_{3}(X, L) \geq-1$. However, we have $A_{3}(X, L) \leq 4$ by employing inequality (15). Thus, one can see that

$$
\left(A_{4}(X, L), A_{3}(X, L)\right)=(1,-1),(1,0),(1,1),(1,2),(1,3) \text { or }(1,4) .
$$

(ii.3.6.1) We consider the case that $\left(A_{4}(X, L), A_{3}(X, L)\right)=(1,-1)$ (respectively $(1,0),(1,1))$. Then, we observe that

$$
\begin{aligned}
h^{0}\left(K_{X}+3 L\right) & =A_{5}(X, L)+2 A_{4}(X, L)+A_{3}(X, L) \\
& =2 A_{4}(X, L)+3 A_{3}(X, L) \\
& = \begin{cases}1, & \text { if } A_{3}(X, L)=-1, \\
2, & \text { if } A_{3}(X, L)=0, \\
3, & \text { if } A_{3}(X, L)=1 .\end{cases}
\end{aligned}
$$


Since $h^{0}(L)>0$, it follows from (34) that

$$
h^{0}\left(K_{X}+4 L\right) \geq \begin{cases}1, & \text { if } A_{3}(X, L)=-1, \\ 2, & \text { if } A_{3}(X, L)=0 \\ 3, & \text { if } A_{3}(X, L)=1\end{cases}
$$

Besides, since

$$
h^{0}\left(K_{X}+4 L\right)=A_{5}(X, L)+3 A_{4}(X, L)+3 A_{3}(X, L)+A_{2}(X, L),
$$

we have the following by employing inequality (35):

$$
A_{2}(X, L) \geq \begin{cases}1, & \text { if } A_{3}(X, L)=-1, \\ -1, & \text { if } A_{3}(X, L)=0 \\ -3, & \text { if } A_{3}(X, L)=1\end{cases}
$$

Therefore, it follows that

$$
\begin{aligned}
h^{0}\left(K_{X}+7 L\right)= & A_{5}(X, L)+6 A_{4}(X, L)+15 A_{3}(X, L) \\
& +20 A_{2}(X, L)+15 A_{1}(X, L)+6 A_{0}(X, L) \\
> & 7 .
\end{aligned}
$$

(ii.3.6.2) Let us consider the case that $\left(A_{4}(X, L), A_{3}(X, L)\right)=(1,2)$. Then, we observe that

$$
\begin{aligned}
h^{0}\left(K_{X}+3 L\right) & =A_{5}(X, L)+2 A_{4}(X, L)+A_{3}(X, L) \\
& =2 A_{4}(X, L)+3 A_{3}(X, L) \\
& =4 .
\end{aligned}
$$

Since $h^{0}(L)>0$, we get from (36) that

$$
h^{0}\left(K_{X}+4 L\right) \geq 4 .
$$

Since

$$
h^{0}\left(K_{X}+4 L\right)=A_{5}(X, L)+3 A_{4}(X, L)+3 A_{3}(X, L)+A_{2}(X, L),
$$

we have $A_{2}(X, L) \geq-5$ by utilizing inequality (37).

Here, we assume that $7>h^{0}\left(K_{X}+7 L\right)$. Then, using (10), Claim 3.3 and assumptions, we get

$$
\begin{aligned}
7 & >h^{0}\left(K_{X}+7 L\right) \\
& =A_{5}(X, L)+6 A_{4}(X, L)+15 A_{3}(X, L)+20 A_{2}(X, L)+15 A_{1}(X, L)+6 A_{0}(X, L) \\
& \geq 20 A_{2}(X, L)+87 .
\end{aligned}
$$

It follows that

$$
A_{2}(X, L) \leq-5 \text {. }
$$

Therefore, we have $A_{2}(X, L)=-5$. 
Assume that $A_{2}(X, L)=-5$. Since $h^{0}(L)>0$, we observe from inequality (37) that

$$
h^{0}\left(K_{X}+5 L\right) \geq 4 .
$$

Thus, we get from inequality (38) and (32) that $A_{1}(X, L) \geq 8$. Therefore, it follows that

$$
\begin{aligned}
h^{0}\left(K_{X}+7 L\right)= & A_{5}(X, L)+6 A_{4}(X, L)+15 A_{3}(X, L) \\
& +20 A_{2}(X, L)+15 A_{1}(X, L)+6 A_{0}(X, L) \\
> & 7 .
\end{aligned}
$$

This contradicts the assumption that $h^{0}\left(K_{X}+7 L\right)<7$.

(ii.3.6.3) We consider the case that $\left(A_{4}(X, L), A_{3}(X, L)\right)=(1,3)$. Then, we obtain that

$$
\begin{aligned}
h^{0}\left(K_{X}+3 L\right) & =A_{5}(X, L)+2 A_{4}(X, L)+A_{3}(X, L) \\
& =2 A_{4}(X, L)+3 A_{3}(X, L) \\
& =5 .
\end{aligned}
$$

Using $h^{0}(L)>0$, we obtain from (39) that

$$
h^{0}\left(K_{X}+4 L\right) \geq 5 .
$$

Since

$$
h^{0}\left(K_{X}+4 L\right)=A_{5}(X, L)+3 A_{4}(X, L)+3 A_{3}(X, L)+A_{2}(X, L),
$$

we have $A_{2}(X, L) \geq-7$ by applying inequality (40).

Here, we assume that $7>h^{0}\left(K_{X}+7 L\right)$. Then, by employing equation (10), Claim 3.3 and assumptions, we have

$$
\begin{aligned}
7 & >h^{0}\left(K_{X}+7 L\right) \\
& =A_{5}(X, L)+6 A_{4}(X, L)+15 A_{3}(X, L)+20 A_{2}(X, L)+15 A_{1}(X, L)+6 A_{0}(X, L) \\
& \geq 20 A_{2}(X, L)+102 .
\end{aligned}
$$

It follows that

$$
A_{2}(X, L) \leq-5 \text {. }
$$

Therefore, $A_{2}(X, L)=-5,-6$ or -7 . Consequently, one can see that

$$
\begin{aligned}
h^{0}\left(K_{X}+4 L\right) & =A_{5}(X, L)+3 A_{4}(X, L)+3 A_{3}(X, L)+A_{2}(X, L) \\
& = \begin{cases}7, & \text { if } A_{2}(X, L)=-5, \\
6, & \text { if } A_{2}(X, L)=-6, \\
5, & \text { if } A_{2}(X, L)=-7 .\end{cases}
\end{aligned}
$$

By employing $h^{0}(L)>0$, we obtain from equation (41) that

$$
h^{0}\left(K_{X}+5 L\right) \geq \begin{cases}7, & \text { if } A_{2}(X, L)=-5 \\ 6, & \text { if } A_{2}(X, L)=-6 \\ 5, & \text { if } A_{2}(X, L)=-7\end{cases}
$$


Hence, we get from inequality (42) and (32) that

$$
A_{1}(X, L) \geq \begin{cases}5, & \text { if } A_{2}(X, L)=-5 \\ 8, & \text { if } A_{2}(X, L)=-6 \\ 11, & \text { if } A_{2}(X, L)=-7\end{cases}
$$

Therefore, we obtain that

$$
\begin{aligned}
h^{0}\left(K_{X}+7 L\right)= & A_{5}(X, L)+6 A_{4}(X, L)+15 A_{3}(X, L) \\
& +20 A_{2}(X, L)+15 A_{1}(X, L)+6 A_{0}(X, L) \\
> & 7 .
\end{aligned}
$$

This contradicts the assumption that $h^{0}\left(K_{X}+7 L\right)<7$.

(ii.3.6.4) Let us consider the case that $\left(A_{4}(X, L), A_{3}(X, L)\right)=(1,4)$. Then, we observe that

$$
\begin{aligned}
h^{0}\left(K_{X}+3 L\right) & =A_{5}(X, L)+2 A_{4}(X, L)+A_{3}(X, L) \\
& =2 A_{4}(X, L)+3 A_{3}(X, L) \\
& =6 .
\end{aligned}
$$

Since $h^{0}(L)>0$, it follows from (43) that

$$
h^{0}\left(K_{X}+4 L\right) \geq 6 .
$$

Meanwhile, since

$$
h^{0}\left(K_{X}+4 L\right)=A_{5}(X, L)+3 A_{4}(X, L)+3 A_{3}(X, L)+A_{2}(X, L),
$$

we have $A_{2}(X, L) \geq-9$ by applying inequality (44).

Here, we assume that $7>h^{0}\left(K_{X}+7 L\right)$. Then, using equation (10), Claim 3.3 and assumptions, we have

$$
\begin{aligned}
7 & >h^{0}\left(K_{X}+7 L\right) \\
& =A_{5}(X, L)+6 A_{4}(X, L)+15 A_{3}(X, L)+20 A_{2}(X, L)+15 A_{1}(X, L)+6 A_{0}(X, L) \\
& \geq 20 A_{2}(X, L)+117 .
\end{aligned}
$$

It follows that

$$
A_{2}(X, L) \leq-6 .
$$

Therefore $A_{2}(X, L)=-6,-7,-8$ or -9 .

Assume that $A_{2}(X, L)=-6$ (respectively $\left.-7,-8,-9\right)$. Then, we get

$$
\begin{aligned}
h^{0}\left(K_{X}+4 L\right) & =A_{5}(X, L)+3 A_{4}(X, L)+3 A_{3}(X, L)+A_{2}(X, L) \\
& = \begin{cases}9, & \text { if } A_{2}(X, L)=-6, \\
8, & \text { if } A_{2}(X, L)=-7, \\
7, & \text { if } A_{2}(X, L)=-8, \\
6, & \text { if } A_{2}(X, L)=-9 .\end{cases}
\end{aligned}
$$


Since $h^{0}(L)>0$, we obtain from equation (45) that

$$
h^{0}\left(K_{X}+5 L\right) \geq \begin{cases}9, & \text { if } A_{2}(X, L)=-6 \\ 8, & \text { if } A_{2}(X, L)=-7 \\ 7, & \text { if } A_{2}(X, L)=-8 \\ 6, & \text { if } A_{2}(X, L)=-9\end{cases}
$$

Thus, we see from inequality (46) and (32) that

$$
A_{1}(X, L) \geq \begin{cases}5, & \text { if } A_{2}(X, L)=-6 \\ 8, & \text { if } A_{2}(X, L)=-7 \\ 11, & \text { if } A_{2}(X, L)=-8 \\ 14, & \text { if } A_{2}(X, L)=-9\end{cases}
$$

Therefore, we have

$$
\begin{aligned}
h^{0}\left(K_{X}+7 L\right)= & A_{5}(X, L)+6 A_{4}(X, L)+15 A_{3}(X, L) \\
& +20 A_{2}(X, L)+15 A_{1}(X, L)+6 A_{0}(X, L) \\
> & 7 .
\end{aligned}
$$

This contradicts the assumption that $h^{0}\left(K_{X}+7 L\right)<7$.

(ii.4) We consider the case that $m=8$. Assume that $h^{0}\left(K_{X}+8 L\right)<22$. Since $h^{0}\left(K_{X}+\right.$ $7 L) \geq 7$, we have $h^{0}\left(K_{X}+8 L\right)-h^{0}\left(K_{X}+7 L\right)<15$. Moreover,

$$
\begin{aligned}
& h^{0}\left(K_{X}+8 L\right)-h^{0}\left(K_{X}+7 L\right) \\
& \quad=A_{4}(X, L)+6 A_{3}(X, L)+15 A_{2}(X, L)+20 A_{1}(X, L)+15 A_{0}(X, L) .
\end{aligned}
$$

Therefore, we get

$$
A_{4}(X, L)+6 A_{3}(X, L)+15 A_{2}(X, L)+20 A_{1}(X, L)+15\left(A_{0}(X, L)-1\right)<0 .
$$

Next, we evaluate the left-hand side of this inequality. First, we note that $A_{4}(X, L) \geq 0$. Moreover, we observe that

$$
\begin{aligned}
6 A_{3}(X, L)+15 A_{2}(X, L)+20 A_{1}(X, L)+15\left(A_{0}(X, L)-1\right) \\
=9 L^{5}+\frac{25}{4} K_{X} L^{4}+K_{X}^{2} L^{3}+\frac{1}{4} c_{2}(X)\left(K_{X}+4 L\right) L^{2}+\frac{195}{4} L^{5} \\
\quad+\frac{75}{4} K_{X} L^{4}+\frac{5}{4}\left(K_{X}^{2}+c_{2}(X)\right) L^{3}+10 K_{X} L^{4}+60 L^{5}+15 L^{5}-15 \\
=\frac{471}{4} L^{5}+35 K_{X} L^{4}+\frac{9}{4} K_{X}^{2} L^{3}+\frac{1}{4} c_{2}(X)\left(K_{X}+9 L\right) L^{2}+15 L^{5}-15 .
\end{aligned}
$$

The application of inequality (7) gives that

$$
\begin{aligned}
\frac{1}{4} c_{2}(X)\left(K_{X}+9 L\right) L^{2} & \geq-\frac{1}{4}\left(K_{X}+9 L\right) L^{2}\left(8 K_{X} L+40 L^{2}\right) \\
& =-2 K_{X}^{2} L^{3}-28 K_{X} L^{4}-90 L^{5} .
\end{aligned}
$$


Thus, by employing equation (48) and inequality (49), we have

$$
\begin{aligned}
& 6 A_{3}(X, L)+15 A_{2}(X, L)+20 A_{1}(X, L)+15\left(A_{0}(X, L)-1\right) \\
& \quad \geq \frac{1}{4} K_{X}^{2} L^{3}+7 K_{X} L^{4}+\frac{111}{4} L^{5}+15\left(L^{5}-1\right) \\
& \quad=\frac{1}{4}\left(K_{X}+26 L\right)\left(K_{X}+2 L\right)+\frac{59}{4} L^{5}+15\left(L^{5}-1\right) \\
& \quad \geq 1 .
\end{aligned}
$$

This contradicts inequality (47). Hence, we obtain the assertion of Proposition 3.2.

(iii) Let us consider the case that $h^{0}\left(K_{X}+L\right)>0$. If we can show that $3 A_{3}(X, L)+(m-$ 3) $A_{2}(X, L) \geq 0$ using Claim 3.2 and inequality (3), then we have that $h^{0}\left(K_{X}+m L\right)>$ $\left(\begin{array}{c}m-1 \\ 5\end{array}\right)$ holds. By employing Remark 2.2(iii), we get

$$
\begin{aligned}
& 3 A_{3}(X, L)+(m-3) A_{2}(X, L) \\
& =\frac{13 m-21}{4} L^{5}+\frac{10 m-5}{8} K_{X} L^{4}+\frac{m+3}{12} K_{X}^{2} L^{3} \\
& \quad+\frac{3 K_{X}+(2 m+6) L}{24} c_{2}(X) L^{2} .
\end{aligned}
$$

However, using Proposition 2.1 and the assumption that $h^{0}\left(K_{X}+L\right)>0$, we infer that $\Omega_{X}\langle L\rangle$ is generically nef. Furthermore, we observe that $K_{X}+5 L$ is ample since we assumed that $K_{X}+4 L$ is nef. Hence, by utilizing the generical nefness of $\Omega_{X}\langle L\rangle$ and $[\mathbf{1 8}, 2.11$ Corollary], we have

$$
\begin{aligned}
& \frac{3 K_{X}+(2 m+6) L}{24} c_{2}(X) L^{2} \\
& \geq-\frac{1}{24}\left(12 K_{X}^{2}+(8 m+54) K_{X} L+(20 m+60) L^{2}\right) L^{3} .
\end{aligned}
$$

Thus, by applying (50) and inequality (51) we get

$$
\begin{aligned}
& 3 A_{3}(X, L)+(m-3) A_{2}(X, L) \\
& \geq \frac{m-3}{12} K_{X}^{2} L^{3}+\frac{22 m-69}{24} K_{X} L^{4}+\frac{58 m-186}{24} L^{5} \\
& =\frac{m-3}{24} L^{3}\left(2 K_{X}^{2} L^{3}+\frac{22 m-69}{m-3} K_{X} L+\frac{58 m-186}{m-3} L^{2}\right) \\
& =\frac{m-3}{24} L^{3}\left(\left(2 K_{X}+14 L\right)\left(K_{X}+3 L\right)+\frac{2 m-9}{m-3}\left(K_{X}+8 L\right) L+\frac{2 m+12}{m-3} L^{2}\right) \\
& \geq 0 .
\end{aligned}
$$

Therefore, we obtain that $h^{0}\left(K_{X}+m L\right)>\left(\begin{array}{c}m-1 \\ 5\end{array}\right)$ for case (iii).

Hence, using cases (i), (ii) and (iii), we get the assertion of Theorem 3.1.

Acknowledgements. This research was supported by JSPS KAKENHI Grant Number $16 \mathrm{~K} 05103$. 


\title{
REFERENCES
}

[1] M. Andreatta and C. Fontanari. Effective adjunction theory. Ann. Univ. Ferrara 64 (2018), 243-257.

[2] M. C. Beltrametti and A. J. Sommese. The Adjunction Theory of Complex Projective Varieties (de Gruyter Expositions in Mathematics, 16). Walter de Gruyter, Berlin, 1995.

[3] A. Broustet. Non-annulation effective et positivité locale des fibrés en droites amples adjoints. Math. Ann. 343 (2009), 727-755.

[4] A. Broustet and A. Höring. Effective non-vanishing conjectures for projective threefolds. Adv. Geom. 10 (2010), 737-746.

[5] F. Campana. Connexité rationnelle des variétés de Fano. Ann. Sci. Éc. Norm. Supér. (4) 25 (1992), 539-545.

[6] L. Ein and R. Lazarsfeld. Global generation of pluricanonical and adjoint linear series on smooth projective threefolds. J. Amer. Math. Soc. 6 (1993), 875-903.

[7] T. Fujita. Classification Theories of Polarized Varieties (London Mathematical Society Lecture Note Series, 155). Cambridge University Press, Cambridge, 1990.

[8] Y. Fukuma. A lower bound for sectional genus of quasi-polarized manifolds. J. Math. Soc. Japan 49 (1997), 339-362.

[9] Y. Fukuma. On the sectional geometric genus of quasi-polarized varieties, I. Comm. Algebra 32 (2004), 1069-1100.

[10] Y. Fukuma. A formula for the sectional geometric genus of quasi-polarized manifolds by using intersection numbers. J. Pure Appl. Algebra 194 (2004), 113-126.

[11] Y. Fukuma. On a conjecture of Beltrametti-Sommese for polarized 3-folds. Internat. J. Math. 17 (2006), 761-789.

[12] Y. Fukuma. A study on the dimension of global sections of adjoint bundles for polarized manifolds. J. Algebra 320 (2008), 3543-3558.

[13] Y. Fukuma. On classification of polarized 3-folds $(X, L)$ with $h^{0}\left(K_{X}+2 L\right)=2$. Beiträge Algebra Geom. 55 (2014), 77-103.

[14] Y. Fukuma. On a conjecture of Beltrametti-Sommese for polarized 4-folds. Kodai Math. J. 38 (2015), 343-351.

[15] Y. Fukuma. On polarized 4-folds $(X, L)$ with $h^{0}\left(K_{X}+3 L\right)=1$. J. Pure Appl. Algebra 220 (2016), 1178-1187.

[16] Y. Fukuma. On the dimension of $H^{0}\left(K_{X}+m L\right)$ of polarized $n$-folds $(X, L)$ with $m \geq n$. Kyushu J. Math. 71 (2017), 115-128.

[17] T. Graber, J. Harris and J. Starr. Families of rationally connected varieties. J. Amer. Math. Soc. 16 (2003), 57-67.

[18] A. Höring. On a conjecture of Beltrametti and Sommese. J. Algebraic Geom. 21 (2012), 721-751.

[19] Y. Kawamata. On Fujita's freeness conjecture for 3-folds and 4-folds. Math. Ann. 308 (1997), 491-505.

[20] J. Kollár. Shafarevich Maps and Automorphic Forms (M. B. Porter Lectures). Princeton University Press, Princeton, 1995.

[21] J. Kollár, Y. Miyaoka and S. Mori. Rational connectedness and boundedness of Fano manifolds. J. Differential Geom. 36 (1992), 765-779.

[22] I. Reider. Vector bundles of rank 2 and linear systems on algebraic surfaces. Ann. of Math. (2) 127 (1988), 309-316.

[23] F. Ye and Z. Zhu. Global generation of adjoint line bundles on projective 5-folds. Manuscripta Math. 153 (2017), 545-562.

\author{
Yoshiaki Fukuma \\ Department of Mathematics and Physics \\ Faculty of Science and Technology \\ Kochi University \\ Akebono-cho, Kochi 780-8520 \\ Japan \\ (E-mail: fukuma@kochi-u.ac.jp)
}

